\title{
CORRESPONDENCE.
}

\section{ON THE ADJUSTMENT OF MORTALITY TABLES.}

To the Editor of the Journal of the Institute of Actuaries.

Sir,-The important problem of adjusting Mortality Tables has lately been discussed by Dr. Kanner, and I hope the following statement of his theory will not be deroid of interest to the reader's of this periodical. The publication of this speculation was the last work of Dr. Kanner, who suddenly died of small-pox in the beginning of this month at Berlin, in the midst of his assiduous life.

Dr. Kanner introduced his theme by the following problem (published in No. 16 of the Annalen des gesammten Versicherungswesens). Assuming that it has been observed that out of 994 persons of a given age 4 have died in the course of a year, and that out of 3012 persons one year older, 72 have died in the course of a year: further, that we have reason to presume that the chance of dying in a year at the higher age exceeds that at the lower, by 01: what value must be attributed to the chance of dying in a year at the younger age in order to make the mortality really observed at the two ages the most probable? Or, in other words, what is the most probable mortality for each of the two ages, to be deduced from the observations, under the condition that the probability of dying in a year is $\cdot 01$ greater at the higher age than at the younger?

Let $x_{0}$ be the probability of dying in a year at the younger age, then $x_{0}+\cdot 01$ is the same probability at the higher age; and according to well known rules of the theory of probabilities, the probability of the hypothesis that the mortality of the younger age is $x_{0}$ becomes proportional to

$$
\frac{\left(1-x_{0}\right)^{990} x_{0}^{4}\left\{1-\left(x_{0}+\cdot 01\right)\right\}^{2940}\left(x_{0}+\cdot 01\right)^{72}}{\int_{0}^{1}(1-x)^{990} x^{4} d x \int_{0}^{1}(1-x)^{2940} x^{72} d x} .
$$

This becomes a maximum when the uumerator is a maximum; and then we have

$\mathrm{Ol}^{*}$

$$
\begin{gathered}
\frac{d}{d x_{0}}\left\{\left(1-x_{0}\right)^{290} x_{0}^{4}\left(\cdot 99-x_{0}\right)^{2940}\left(x_{0}+\cdot 01\right)^{72}\right\}=0 \\
-\frac{990}{1-x_{0}}+\frac{4}{x_{0}}-\frac{2940}{99-x_{0}}+\frac{72}{01+x_{0}}=0,
\end{gathered}
$$

whence we get

$$
4006 x_{0}{ }^{3}-4032 x_{0}{ }^{2}+35 \cdot 9594 x_{0}+\cdot 0396=0 .
$$

The only real root of this equation of the third degree is $x_{0}=\cdot 01$;

therefore the rates of mortality,

01 for the younger age,

.02 for the higher age,

are the sought most probable rates corresponding to the two observations, under the condition that the rate at the higher age exceeds that at the younger by $\cdot 01$.

In Number 17 of the Annalen Dr. Kanner gives the development of 
the theory in general, after having pointed out that the absence of a clear' perception of the problem had induced mathematicians almost to consider it insoluble. They left the adjustment of a Mortality Table to esthetic taste. What abstract thought was not able to perform, the eye and the feeling were to decide, by the aid of a graphic exhibition of the numbers!

Dr. Kanner conceives the problem in the following way:

Given the numbers of observed persons of different ages and the numbers of them who die in the course of a year;

Required the most probable mortality for each age.

If there are given no other conditions, which the mortalities of the different ages are to satisfy, an unadjusted Mortality Table is wanted. If, on the contrary, the rates of mortality at the different ages are to depend on each other and satisfy certain conditions, expressed by equations, the rates are made subject to a law, arbitrary, but answering to experience; and an adjusted Table of Mortality is to be computed.

The first of these problems may be considered as a special case of the second.

Let $\quad m_{0}, m_{1}, m_{2}, \ldots m_{\mu}$,

denote the numbers of persons dying in a year, at $\mu+1$ ages,

$$
n_{0}, n_{1}, n_{2}, \ldots n_{\mu}
$$

the numbers of persons of the same ages who survive the year, these. numbers having been found by careful observation.

Let

$$
x_{0}, x_{1}, x_{2}, \ldots x_{\mu},
$$

denote the rates of mortality at the different ages. It is required to find their most probable values.

Evidently the solution of the problem requires that the product

$$
x_{0}^{m_{0}}\left(1-x_{0}\right)^{n_{0}} x_{1}{ }^{m_{1}}\left(1-x_{1}\right)^{n_{1}} \ldots x_{\mu}{ }^{m_{\mu}}\left(1-x_{\mu}\right)^{n_{\mu}}=\mathrm{V}
$$

must be a maximum. This condition is satisfied by putting its partial differential coefficients equal to 0 .

Thus

$$
\frac{d \mathrm{~V}}{d x_{0}}=0, \frac{d \mathrm{~V}}{d x_{1}}=0, \ldots \frac{d \mathrm{~V}}{d x_{\mu}}=0 \text {. }
$$

In this way, each age being considered separately and independently of the other ages, the most probable rate of mortality at each age is found to be equal to the ratio of the number of persons dying in a year to the number living at the beginning of the year. 'The observation gave us nothing but the proportion of the persons dying out of a certain number living; and therefore it allows no other conclusion but what is the most probable hypothesis of mortality for each different age. The observation does not include any connection or relationship between the single mortalities $x_{0}, x_{1}, \ldots x_{\mu}$, and if the observation is to be the sole basis of our conclusions, we are obliged to consider the rates of mortality $x_{0}, x_{1}, \ldots x_{\mu}$, as independent of each other. By solving the above equations,

$$
\frac{d \mathrm{~V}}{d x_{0}}=0, \frac{d \mathrm{~V}}{d x_{1}}=0, \ldots \frac{d \mathrm{~V}}{d x_{\mu}}=0,
$$


we find

$$
x_{0}=\frac{m_{0}}{m_{0}+n_{0}}, x_{1}=\frac{m_{1}}{m_{1}+n_{1}}, \ldots x_{\mu}=\frac{m_{\mu}}{m_{\mu}+n_{\mu}}
$$

The observation enables us also to state the probability of each of these hypothesises, as well as the probability of any other hypothesis which we may like to make; but there is not the least support for any adjustment, if we intend to proceed on the basis of the observation exclusively. Every adjustment is opposed to the result of observation, and the problem of adjustment cannot be introduced at all until we have established, either tacitly, or explicitly, relations between the unknown rates $x_{0}, x_{1}, \ldots x_{\mu}$, - conditions which these values are to satisfy, and with which the values $\frac{m_{0}}{m_{0}+n_{0}}, \frac{m_{1}}{m_{1}+n_{1}}, \ldots \frac{m_{\mu}}{m_{\mu}+n_{\mu}}$, cannot be reconciled. Only then can we raise the question of adjustment, the question of what values we shall select for $x_{0}, x_{1}, \ldots x_{\mu}$, so as to satisfy the relations in question and at the same time make $\mathrm{V}$ a maximum; for this latter condition is the basis which must be maintained, if we wish to find out the most probable hypothesis to be derived from our observations.

If the values which link $x_{0}, x_{1}, \ldots x_{\mu}$, together are substituted in $\mathrm{V}$, one or more quantities are to be determined in such a way that $\mathrm{V}$ shall be a maximum, and thus the problem of adjustment will be solved.

The most perfect way to express the relations between $x_{0}, x_{1}, \ldots x_{\mu}$ consists in considering $x$ as a function of the age. Thus, if

$$
x=f(t, a, b, c \ldots)
$$

where $t$ is the age, and $a, b, c, \ldots$ are constants to be determined, this may be called the mathematical law of mortality.

If $t_{0}, t_{1}, \ldots t_{\mu}$ are the ages to which the observations refer, we have to determine $a, b, c, \ldots$ in the expression

$$
\begin{aligned}
& \left\{f\left(t_{0}, a, b, c, \ldots\right)\right\}^{n_{0}} \cdot\left\{1-f\left(t_{0}, a, b, c, \ldots\right)\right\}^{n_{0}} \ldots \ldots \\
& \ldots\left\{f\left(t_{\mu}, a, b, c, \ldots\right)\right\}^{m_{\mu}}\left\{1-f\left(t_{\mu}, a, b, c, \ldots\right)\right\}^{n_{\mu}}=\mathrm{V}
\end{aligned}
$$

so that $\mathrm{V}$ shall be a maximum.

For brevity, write

$$
\mathrm{V}=f_{0}^{m_{0}}\left(1-f_{0}\right)^{n_{0}} f_{\mathrm{l}}^{m_{1}}\left(1-f_{1}\right)^{n_{1}} \ldots f_{\mu}^{m_{\mu}}\left(1-f_{\mu}\right)^{n_{\mu}}
$$

Then forming the partial differential coefficients with respect to $a, b, c, \ldots$ and putting each $=0$, we have

$$
\left.\begin{array}{c}
\frac{m_{0}}{f_{0}} \cdot \frac{d f_{0}}{d a}-\frac{n_{0}}{1-f_{0}} \cdot \frac{d f_{0}}{d a}+\frac{m_{1}}{f_{1}} \cdot \frac{d f_{1}}{d a}-\frac{n_{1}}{1-f_{1}} \cdot \frac{d f_{1}}{d a}+\ldots-\frac{n_{\mu}}{1-f_{\mu}} \cdot \frac{d f_{\mu}}{d a}=0 \\
\frac{m_{0}}{f_{0}} \cdot \frac{d f_{0}}{d b}-\frac{n_{0}}{1-f_{0}} \cdot \frac{d f_{0}}{d b}+\frac{m_{1}}{f_{1}} \cdot \frac{d f_{1}}{d b}-\frac{n_{1}}{1-f_{1}} \cdot \frac{d f_{1}}{d b}+\ldots \ldots-\frac{n_{\mu}}{1-f_{\mu}} \cdot \frac{d f_{\mu}}{d b}=0 \\
\ldots \ldots \ldots \ldots \ldots \ldots \ldots \ldots \ldots \ldots
\end{array}\right\}
$$

-as many equations as there are constants to be determined.

It is not to be denied that the practical application of this theory offers still a great many difficulties, that the form of the last equations is in general so very complicated that we must almost despair of solving them exactly. But these points, at all events, are of secondary importance. It 
may be of importance to find out the way for practical solution of the problem too, but the theory of the question itself had first to be investigated, or else the way which leads to the solution would not have been discernible. In establishing a sound theory, we must never suffer ourselves to contemplate the difficulties that may arise afterwards, when the theory is to be applied for practical purposes; for such a course would darken the accuracy of our conclusions. I see Dr. Kanner's principal merit in his clear proof, that the question of adjustment depends only on the state of relationship which is presumed to exist between the rates of mortality at the different ages, and that the condition that the product $\mathrm{V}$ or $x_{0}{ }^{m_{0}}\left(1-x_{0}\right)^{n_{0}} \mathrm{X}$ $x_{1}^{m_{1}}\left(1-x_{1}\right)^{n_{1}} \ldots x_{\mu}^{m_{\mu}}\left(1-x_{\mu}\right)^{n_{\mu}}$ is to be a maximum, must be made the basis of our conclusions and computations.

Pursuing his examinations, Dr. Kanner mentions the ineffective efforts which have been made to solve the question of adjusting a mortality table by introducing the method of least squares, a method applicable only where the probability of a deviation from the correct value is the same for a positive deviation as for a negative one of the same magnitude, which is not the case in our question.

The general term of the equations $(a)$ is

$$
\frac{m}{f} \cdot \frac{d f}{d a}-\frac{n}{1-f} \cdot \frac{d f}{d a}
$$

which may be put in the form

$$
-\frac{m+n}{2 f(1-f)} 2\left(f-\frac{m}{n+m}\right) \frac{d f}{d a} \text {. }
$$

The incorrect application of the method of least squares, in assuming $\frac{(m+n)^{3}}{2 m n}$ to be the weight of the observations, leads us to the form

$$
\frac{m+n}{2 \cdot \frac{m}{m+n} \cdot \frac{n}{m+n}}: 2\left(f-\frac{m}{n+m}\right) \frac{d f}{d a} \text {. }
$$

Although the two forms resemble each other, their difference is remarkable. If the weight of the observation could be made equal to $\frac{m+n}{2 f(1-f)}$, tho forms would become identical; but I doubt very much whether there exists more than a mere resemblance, and whether the method of least squares can be correctly applied to the problem of adjustment. As Dr. Kanner r emarks, the weight of the observation, in this form, being dependent on the correct values to be found, cannot be determined before the solution of the problem is given.

\section{Hamburg, 30th Nov. 1871.}

$$
\begin{aligned}
& \text { I am, Sir, } \\
& \text { Yours faithfully, }
\end{aligned}
$$

\section{WILHELM LAZARUS.}

** We regret greatly to hear of the death of Dr. Kanner, who has done much to promote sound views in the theory of life contingencies; and whose last production, explained in the foregoing letter, is, altho difficult of practical application, a most valuable contribution to the theory. We consider his premature death a heavy loss to the actuarial profession.-ED. J. I. $\boldsymbol{A}$. 